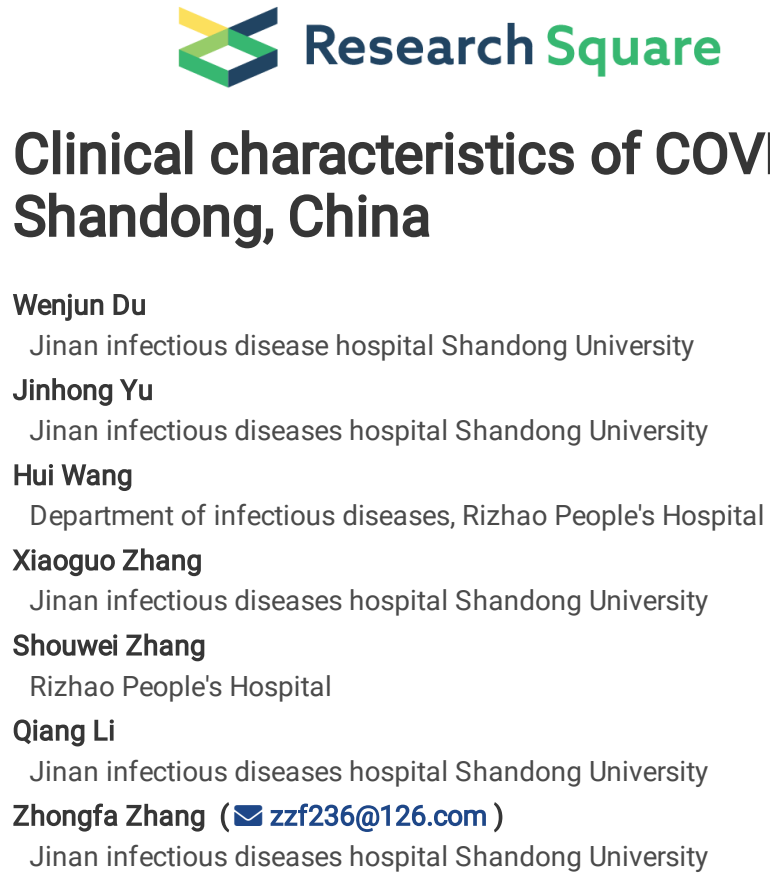

\title{
Clinical characteristics of COVID-19 in children compared with adults in Shandong, China
}




\section{Abstract}

Aims \& Background: The COVID-19 outbreak spread in China and is a threat to the world. We reported on the epidemiological, clinical, laboratory, and radiological characteristics of children cases to help health workers better understand and provide timely diagnosis and treatment.

Methods: Retrospectively, two research centers' case series of 67 consecutive hospitalized cases including 14 children cases with COVID-19 between 23 Jan 2020 to 15 Feb 2020 from Jinan and Rizhao were enrolled in this study. Epidemiological, clinical, laboratory, and radiological characteristics of children and adults were analyzed and compared.

Results: Most cases in children were mild(21.4\%) and conventional cases(78.6\%), with mild clinical signs and symptoms, and all cases were of family clusters. Fever (35.7\%) and dry cough(21.4\%) were described as clinical manifestations in children cases. Dry cough and phlegm were not the most common symptoms in children compared with adults $(\mathrm{p}=0.03)$. In the early stages of the disease, lymphocyte counts did not significantly decline but neutrophils counts did in children compared with adults $(p=0.00)$. There was an elevated level of $\operatorname{LDH}(p=0.01)$ and a lower level of $C R P(p=0.00)$ and IL-6 $(p=0.01)$ in children compared with adults. There were $8(57.1 \%)$ asymptomatic cases and $6(42.9 \%)$ symptomatic cases among the 14 children cases. The age of asymptomatic patients was younger than that of symptomatic patients $(p=0.03)$. Even among asymptomatic patients, $5(62.5 \%)$ cases had pneumonia including $3(60 \%)$ cases with bilateral pneumonia, which was not different compared with that of asymptomatic cases $(p=0.58, p=0.74)$.

Conclusions: The clinical symptoms of children are mild, and the positive indicators of laboratory tests are rare, which may easily cause clinical misdiagnoses.

\section{Introduction}

Since the first atypical pneumonia case was reported in Wuhan, China on December 31, 2019 ${ }^{[1]}$, the pathogen was soon identified ${ }^{[2]}$ and tentatively named 2019-nCoV by the World Health Organization (WHO) ${ }^{[3]}$.On 11 Feb 2020, the World Health Organization officially named it SARS-CoV-2(Severe Acute Respiratory Syndrome Coronavirus-2) and the SARS-CoV-2 infection was named as the COVID-19(Coronavirus Disease 2019) ${ }^{[4]}$.The COVID-19 outbreak quickly spread in Wuhan city and other districts of Hubei Province with a further spread across the Chinese mainland. By 15 Feb 2020 , a total of 68,584confirmed cases including 1,666 deaths were reported in China according to the Chinese Center for Disease Control and Prevention official websites and announcements ${ }^{[5]}$. COVID-19 cases were also reported in Thailand, Japan, Singapore, the Republic of Korea, Hong Kong, Taiwan, the US, and some countries in Europe ${ }^{[6]}$. The outbreak is still on-going.

Previous studies reported the main clinical characteristics of COVID-19. Fever, cough, shortness of breath, muscle ache, confusion, and headache were described as clinical manifestations. Lab tests and chest computed tomographic (CT) scans were also evaluated and the results suggested that decreased lymphocyte counts and bilateral pneumonia were common clinical features, especially in severe cases ${ }^{[7,8]}$.

At present, there is no effective anti-virus drug and vaccine for COVID-19, so early detection and isolation treatment are important to control the progression and spread of the disease. However, the current data mainly come from Hubei Province, especially from Wuhan city. Clinical features, especially in children, have not been reported outside Hubei Province.

In this study, we analyzed and compared the epidemic characteristics and clinical features in children and adults outside Hubei Province. The aims were to help health workers better understanding the clinical features of COVID-19 in children and provide timely diagnosis and treatment.

\section{Methods}

\section{Patients}

A total of 67cases including 14 children cases of confirmed COVID-19 from the Jinan infectious diseases hospital and Rizhao people's hospital, which were the designated hospitals in Jinan and Rizhao city, between 23 Jan 2020 to 15 Feb 2020 were enrolled in this study. The project was approved by the ethics board of Jinan infectious hospital (No.20200203). Informed consent was obtained from each patient or their guardian.All patients enrolled in this study were diagnosed according to the pneumonia diagnosis and treatment plan for the new coronavirus infection formulated by the National Health Commission (trial version 5) ${ }^{[9]}$.Diagnostic criteria for mild cases: mild clinical symptoms, no radiographic findings of pneumonia. Diagnostic criteria for common cases: fever, respiratory symptoms, and radiographic manifestations of pneumonia. Diagnostic criteria for severe cases: (1) respiratory distress, respiratory frequency $\geq 30$ times/min;(2) hypoxemia, with resting oxygen saturation $\leqq 93 \%$;and (3) arterial partial oxygen pressure (PaO2)/oxygen absorption concentration (FiO2) $\leqq 300$ $\mathrm{mmHg}(1 \mathrm{mmHg}=0.133 \mathrm{kPa})$. The diagnostic criteria for critical cases: (1) respiratory failure and mechanical ventilation is required;(2) shock; and (3) complicated with other organ failure requiring ICU care. There are four types of infection:(1) imported cases defined as a history of a sojourn in Hubei Province within 14 days of onset of the illness.(2) Family cluster defined as 3 or more than 3 people with confirmed cases in one family. (3) Close contact defined as close contact with cases from Hubei Province. (4)Unclear; the method of infection is unknown. A child is defined as less than 18 years of age.

\section{Data Collection}

The medical records of patients were analyzed. Information recorded included demographic data, exposure history, symptoms, signs, laboratory findings and chest computed tomographic(CT) scans. Epidemiological, clinical, laboratory, and radiological characteristics data were obtained with data collection forms from electronic medical records. The date of disease onset was defined as the day when the symptom was noticed. 


\section{RTPCR Assay for COVID-19}

Throat swab specimens were collected from patients with suspected COVID-19. COVID-19 was confirmed by RTPCR using the same protocol. The dual-target detection kits were provided by the Shanghai Jienuo Company. Results: the cut-off value was 40 , a Ct value $<37$ was positive, a Ct value $>40$ was negative, and 37-40 was a gray area(the diagnosis needs to be repeated).

\section{Statistical analysis}

Retrieved data were recorded into Microsoft ${ }^{\circledR}$ Excel for Mac (version 16.30) and analyzed. The Statistical Package for Social Sciences version 16.0 (SPSS 16.0;SPSS Inc., Chicago, IL, USA) and the Prism statistical software package(Version 5.0; Graphpad Software Inc. La Jolla, CA, USA) were used. Measurement data were described as mean \pm standard deviation. Background factors were compared using Student's $t$-test (numerical data) or the Chi-square test (categorical data). Differences were regarded as significant if the $p$ value was less than 0.05 on either side.

\section{Results And Findings}

\section{Similar epidemiological and clinical characteristics of cases in Jinan and Rizhao}

A retrospective analysis was performed on patients in Jinan and Rizhao, Shandong Province from 23 Jun to15 Feb 2020 . A total of 67 cases were enrolled in this study. The median age was 34.1 years (range, $0-63$ years), and 32 (47.8\%) were men. Most of cases were mild(11cases, $16.4 \%)$ and conventional (55cases, $82.1 \%)$, with only 1 severe case(1.5\%), which were different from previous reports ${ }^{[7,8]}$ associated with Wuhan city. The most common symptoms at onset of the illness were fever(55.2\%), dry cough(47.8\%), phlegm(31.3\%), and pharyngalgia(29.9\%). Less common symptoms were fatigue(26.9\%), headache(17.9\%), anorexia(11.9\%), myalgia(11.9\%), chest distress( $9 \%)$, nausea(4.5\%), diarrhea(3\%), vomiting (1.5\%), and dizziness (1.5\%). Laboratory tests in most patients showed no significant abnormalities and no significant decreased in lymphocyte counts(6cases, $0.89 \%)$. Of the 67 patients, 56 cases had pneumonia(83.6\%), including 36 bilateral involvements (64.3\%).47 cases in Jinan and 20 cases in Rizhao were analyzed and there was no significant difference in epidemiological characteristics and clinical features except for the proportion of the mild and conventional types $(p=0.01)($ table1).

Table 1. Epidemiological characteristics and Clinical features of COVID-19 in Jinan and Rizhao 


\begin{tabular}{|c|c|c|c|c|}
\hline \multicolumn{5}{|c|}{$\mathrm{N}(\%)$} \\
\hline & Total(67) & $\operatorname{Jinan}(47)$ & Rizhao(20) & $\mathrm{p}$ \\
\hline Age, median, y & 34.10 & 33.04 & 36.60 & 0.47 \\
\hline \multirow[t]{2}{*}{ Gender } & $M(32)(47.8 \%)$ & $\mathrm{M}(20)(42.6 \%)$ & $M(12)(60 \%)$ & 0.19 \\
\hline & $F(35)(52.2 \%)$ & $F(27)(57.4 \%)$ & $F(8)(40 \%)$ & \\
\hline Clinical type: & & & & 0.02 \\
\hline Mild & $11(16.4 \%)$ & $4(8.5 \%)$ & $7(35 \%)$ & \\
\hline Conventional & $55(82.1 \%)$ & $42(89.4 \%)$ & $13(65 \%)$ & \\
\hline Severe & $1(1.5 \%)$ & $1(2.1 \%)$ & $0(0 \%)$ & \\
\hline Critical & $0(0 \%)$ & $0(0 \%)$ & $0(0 \%)$ & \\
\hline Infection method: & & & & 0.16 \\
\hline Imported & $13(19.4 \%)$ & $10(21.3 \%)$ & $3(15 \%)$ & \\
\hline Family cluster & $27(40.3 \%)$ & $20(42.6 \%)$ & $7(35 \%)$ & \\
\hline Close contact & $22(32.8 \%)$ & $12(25.5 \%)$ & $10(50 \%)$ & \\
\hline Unclear & $5(7.5 \%)$ & $5(10.6 \%)$ & $0(0 \%)$ & \\
\hline Time & $7.11 \pm 5.45$ & $6.48 \pm 5.92$ & $8.6 \pm 3.9$ & 0.15 \\
\hline \multicolumn{5}{|c|}{ Signs and symptoms: } \\
\hline Fever & $37(55.2 \%)$ & $27(57.4 \%)$ & $10(50 \%)$ & 0.58 \\
\hline Fever $\left(>=38.5^{\circ} \mathrm{C} \rrbracket\right.$ & $6(16.2 \%)$ & $4(14.8 \%)$ & $2(20 \%)$ & 0.70 \\
\hline Fever $\left(<38.5^{\circ} \mathrm{C}\right)$ & $31(83.8 \%)$ & $23(85.2 \%)$ & $8(80 \%)$ & \\
\hline Dry cough & $32(47.8 \%)$ & $24(51.1 \%)$ & $8(40 \%)$ & 0.41 \\
\hline Phlegm & $21(31.3 \%)$ & $17(36.2 \%)$ & $4(20 \%)$ & 0.19 \\
\hline Headache & $12(17.9 \%)$ & $9(19.1 \%)$ & $3(15 \%)$ & 0.69 \\
\hline Fatigue & $18(26.9 \%)$ & $10(21.3 \%)$ & $8(40 \%)$ & 0.11 \\
\hline Anorexia & $8(11.9 \%)$ & $8(17 \%)$ & $0(0 \%)$ & 0.05 \\
\hline Chest distress & $6(9 \%)$ & $6(12.8 \%)$ & $0(0 \%)$ & 0.09 \\
\hline Dyspnea & $3(4.5 \%)$ & $3(6.4 \%)$ & $0(0 \%)$ & 0.25 \\
\hline Pharyngalgia & $20(29.9 \%)$ & $15(31.9 \%)$ & $5(25 \%)$ & 0.57 \\
\hline Myalgia & $8(11.9 \%)$ & $5(10.6 \%)$ & $3(15 \%)$ & 0.61 \\
\hline Nausea & $3(4.5 \%)$ & $3(6.4 \%)$ & $0(0 \%)$ & 0.25 \\
\hline Vomiting & $1(1.5 \%)$ & $1(2.1 \%)$ & $0(0 \%)$ & 0.51 \\
\hline Diarrhea & $2(3 \%)$ & $2(4.3 \%)$ & $0(0 \%)$ & 0.35 \\
\hline Dizziness & $1(1.5 \%)$ & $1(2.1 \%)$ & $0(0 \%)$ & 0.51 \\
\hline Abdominal pain & $0(0 \%)$ & $0(0 \%)$ & $0(0 \%)$ & \\
\hline Heart rate & $87.80 \pm 12.67$ & $89.33 \pm 13.45$ & $84.35 \pm 10.21$ & 0.15 \\
\hline Respiratory rate & $20.22 \pm 2.70$ & $20.91 \pm 2.58$ & $18.65 \pm 2.32$ & 0.45 \\
\hline
\end{tabular}

Note: Time: from onset to diagnosis

\section{All the cases in children were familial clusters, dry cough and phlegm were not the most common symptoms in children compared with adults}

There were 14 children cases among the 67 cases, with a median age of 6.2 years (range, $0-16 y e a r s)$, and 6 cases(42.9\%)were males. All the cases in children were familial clusters $(\mathrm{p}=0.00)$, with 3 cases $(21.4 \%)$ of the mild type and 11 cases $(78.6 \%)$ of the conventional type and no severe or critical case. Clinical 
symptoms in children were mild or even absent, only 5(35.7\%) cases showed signs of fever, 3 cases(21.4\%) had dry cough, and 1 case had phlegm, Other clinical signs of headache, fatigue, pharyngalgia, and myalgia were rare in children. None of the children developed anorexia, chest distress, dyspnea, nausea, vomiting, diarrhea, dizziness, or abdominal pain. However, dry cough and phlegm were not the most common symptoms in children compared with in adults( $p=0.03$ ). (table2).

Table 2. Epidemiological characteristics and Clinical features of COVID-19 in children and adults

\begin{tabular}{|c|c|c|c|c|}
\hline \multicolumn{5}{|c|}{$N(\%)$} \\
\hline & Total(67) & Children(14) & Adults(53) & $\mathrm{p}$ \\
\hline Age, median, y & 34.10 & 6.20 & 41.47 & 0.00 \\
\hline \multirow[t]{2}{*}{ Gender } & $M(32)(47.8 \%)$ & $M(6)(42.9 \%)$ & $M(26)(49.1 \%)$ & 0.68 \\
\hline & $F(35)(52.2 \%)$ & $F(8)(57.1 \%)$ & $F(27)(50.9 \%)$ & \\
\hline Clinical type: & 67 & 14 & 53 & 0.50 \\
\hline Mild & $11(16.4 \%)$ & $3(21.4 \%)$ & $8(15.1 \%)$ & \\
\hline Conventional & $55(82.1 \%)$ & $11(78.6 \%)$ & $44(83 \%)$ & \\
\hline Severe & $1(1.5 \%)$ & $0(0 \%)$ & $1(1.9 \%)$ & \\
\hline Critical & $0(0 \%)$ & $0(0 \%)$ & $0(0 \%)$ & \\
\hline Infection method: & 67 & 14 & 53 & 0.00 \\
\hline Imported & $13(19.4 \%)$ & $0(0 \%)$ & $13(24.5 \%)$ & \\
\hline Family cluster & $27(40.3 \%)$ & $14(100 \%)$ & $13(24.5 \%)$ & \\
\hline Close contact & $22(32.8 \%)$ & $0(100 \%)$ & $22(41.5 \%)$ & \\
\hline Unclear & $5(7.5 \%)$ & $0(100 \%)$ & $5(9.4 \%)$ & \\
\hline Time & $7.11 \pm 5.45$ & $6.18 \pm 6.41$ & $7.36 \pm 5.21$ & 0.48 \\
\hline \multicolumn{5}{|c|}{ Signs and symptoms: } \\
\hline Fever & $37(55.2 \%)$ & $5(35.7 \%)$ & $32(60.4 \%)$ & 0.10 \\
\hline Fever $\left(>=38.5^{\circ} \mathrm{C} \nabla\right.$ & $6(16.2 \%)$ & $1(20 \%)$ & $5(15.6 \%)$ & 0.81 \\
\hline Fever $\left(<38.5^{\circ} \mathrm{C}\right)$ & $31(83.8 \%)$ & $4(80 \%)$ & $27(84.4 \%)$ & \\
\hline Dry cough & $32(47.8 \%)$ & $3(21.4 \%)$ & $29(54.7 \%)$ & 0.03 \\
\hline Phlegm & $21(31.3 \%)$ & $1(7.1 \%)$ & $20(37.7 \%)$ & 0.03 \\
\hline Headache & $12(17.9 \%)$ & $1(7.1 \%)$ & $11(20.8 \%)$ & 0.24 \\
\hline Fatigue & $18(26.9 \%)$ & $1(7.1 \%)$ & $17(32.1 \%)$ & 0.06 \\
\hline Anorexia & $8(11.9 \%)$ & $0(0 \%)$ & $8(15.1 \%)$ & 0.12 \\
\hline Chest distress & $6(9 \%)$ & $0(0 \%)$ & $6(11.3 \%)$ & 0.19 \\
\hline Dyspnea & $3(4.5 \%)$ & $0(0 \%)$ & $3(5.7 \%)$ & 0.36 \\
\hline Pharyngalgia & $20(29.9 \%)$ & $1(7.1 \%)$ & 19(35.8\%) & 0.04 \\
\hline Myalgia & $8(11.9 \%)$ & $1(7.1 \%)$ & $7(13.2 \%)$ & 0.53 \\
\hline Nausea & $3(4.5 \%)$ & $00(0 \%)$ & $3(5.7 \%)$ & 0.36 \\
\hline Vomiting & $1(1.5 \%)$ & $00(0 \%)$ & $1(1.9 \%)$ & 0.61 \\
\hline Diarrhea & $2(3 \%)$ & $00(0 \%)$ & $2(3.8 \%)$ & 0.46 \\
\hline Dizziness & $1(1.5 \%)$ & $00(0 \%)$ & $1(1.9 \%)$ & 0.61 \\
\hline Abdominal pain & $0(0 \%)$ & $00(0 \%)$ & $0(0 \%)$ & \\
\hline Heart rate & $87.80 \pm 12.67$ & $93.00 \pm 9.99$ & $86.62 \pm 13.00$ & 0.12 \\
\hline Respiratory rate & $20.22 \pm 2.70$ & $21.67 \pm 1.61$ & $19.89 \pm 2.79$ & 0.01 \\
\hline
\end{tabular}

Note: Time: from onset to diagnosis 


\section{There was an elevated LDH value and lower level of CRP in children compared to adults with COVID-19}

Laboratory tests of children and adults were analyzed. The results showed that the white blood cell counts of children were all normal, with decreased neutrophil counts $(p=0.00)$ and increased lymphocyte counts $(p=0.00)$ compared with adults(table3). Considering the physical characteristics of the children, we further analyzed the difference in blood cell counts between children over 6years and adults. Lymphocyte counts showed no difference between the two groups, however, neutrophil counts decreased in both over 6 years $(\mathrm{p}=0.02)$ and less than 6 years in the children group compared with adults(table4). The abnormal rate of white blood cell counts, neutrophil counts, and lymphocyte counts were also evaluated, and the results showed no difference in children compared with adults(table5). COVID-19 caused liver function damage, cardiac muscle damage, and coagulation function changes. The abnormal rate of ALT, AST, LDH, CK, Myo, PT, and D-dimer was 17\%, 7.5\%, 18.9\%, 15.1\%, 3.8\%, 13.2\%, and $35.8 \%$ in adults and $7.7 \%, 7.7 \%, 50 \%, 28.6 \%, 0 \%, 7.1 \%$, and $35.7 \%$ in children, respectively(table5). The value and positive rate of LDH in children were more significantly increased than in adults, which suggested that children are more likely to suffer myocardial damage than adults $(p=0.01, p=0.02)$ (table3,table5). According to evaluation of infection indicators, the value of PCT showed no difference between children and adults, However, the value and positive rate of CRP and the level of IL-6were more significantly increased in adults than in children $(p=0.00, p=0.02, p=0.01$ )(table3, table5). Of the 67 cases, 56 cases had complications of pneumonia(83.6\%) including 11 children and 45 adults. A total of 6 children(64.5\%) and 30 adults(66.7\%) had pneumonia with bilateral involvement, with no difference between the two groups( $p=0.45)$. However, pneumonia in adults was more severe compared with that in children(table5, fig1).

Table 3. Lab tests and CT scan of COVID-19 in children and adults.

\begin{tabular}{|c|c|c|c|c|c|}
\hline \multicolumn{6}{|c|}{ Median (IQR) } \\
\hline & Normal Range & Total $(\mathrm{N}=67)$ & Children $(n=14)$ & Adults $(\mathrm{n}=53)$ & $\mathrm{p}$ \\
\hline WBC $\left(\times 10^{9} / \mathrm{L}\right)$ & $4-10$ & $5.33 \pm 1.84$ & $5.93 \pm 2.52$ & $5.17 \pm 1.60$ & 0.30 \\
\hline $\mathrm{N}\left(\times 10^{9} / \mathrm{L}\right)$ & $2-7$ & $2.88 \pm 1.45$ & $1.76 \pm 0.53$ & $3.18 \pm 1.45$ & 0.00 \\
\hline$L\left(\times 10^{9} / L\right)$ & $0.8-4$ & $2.02 \pm 1.36$ & $3.68 \pm 2.04$ & $1.57 \pm 0.59$ & 0.00 \\
\hline $\mathrm{M}\left(\times 10^{9} / \mathrm{L}\right)$ & $0.12-0.8$ & $0.36 \pm 0.15$ & $0.36 \pm 0.11$ & $0.36 \pm 0.16$ & 0.95 \\
\hline $\mathrm{HBG}(\mathrm{g} / \mathrm{L})$ & $110-150$ & $137.3 \pm 20.02$ & $131.64 \pm 12.92$ & $138.83 \pm 21.38$ & 0.12 \\
\hline $\operatorname{PLT}\left(\times 10^{9} / \mathrm{L}\right)$ & $100-300$ & $213.4 \pm 64.62$ & $243.79 \pm 76.16$ & $205.29 \pm 59.36$ & 0.047 \\
\hline $\mathrm{CRP}(\mathrm{mg} / \mathrm{L})$ & $0.068-8.2$ & $9.05 \pm 15.81$ & $1.26 \pm 2.82$ & $11.19 \pm 17.21$ & 0.00 \\
\hline PCT(ug/L) & $0-0.05$ & $0.05 \pm 0.05$ & $0.05 \pm 0.02$ & $0.05 \pm 0.06$ & 0.83 \\
\hline $\mathrm{LDH}(\mathrm{U} / \mathrm{L})$ & $109-245$ & $222.6 \pm 71.68$ & $298.63 \pm 92.95$ & $205.57 \pm 53.80$ & 0.01 \\
\hline $\mathrm{CK}(\mathrm{U} / \mathrm{L})$ & $26-140$ & $94.38 \pm 57.11$ & $107.54 \pm 44.70$ & $91.08 \pm 59.73$ & 0.36 \\
\hline Myo(ug/L) & $10-46$ & $18.84 \pm 11.61$ & $15.33 \pm 7.92$ & $19.50 \pm 12.13$ & 0.33 \\
\hline Ctn(ug/L) & $0-0.01$ & $0.01 \pm 0.00$ & $0.01 \pm 0.00$ & $0.01 \pm 0.00$ & 0.68 \\
\hline $\mathrm{BUN}(\mathrm{mmol} / \mathrm{L})$ & $2.9-8.2$ & $4.07 \pm 1.21$ & $4.23 \pm 1.12$ & $4.03 \pm 1.24$ & 0.58 \\
\hline $\mathrm{Cr}$ (umol/L) & $50.4-98.1$ & $59.80 \pm 14.29$ & $43.08 \pm 11.20$ & $64.30 \pm 11.44$ & 0.00 \\
\hline PT(s) & 8.8-13.8 & $12.61 \pm 0.99$ & $12.53 \pm 0.89$ & $12.63 \pm 1.01$ & 0.75 \\
\hline D-dimer(ug/ml) & $0-0.5$ & $0.49 \pm 0.37$ & $0.47 \pm 0.26$ & $0.50 \pm 0.40$ & 0.82 \\
\hline $\operatorname{ALT}(\mathrm{U} / \mathrm{L})$ & $-0-40$ & $28.42 \pm 28.31$ & $21.29 \pm 29.18$ & $30.35 \pm 28.05$ & 0.291 \\
\hline AST(U/L) & $0-40$ & $25.47 \pm 12.77$ & $29.00 \pm 8.84$ & $24.52 \pm 13.54$ & 0.25 \\
\hline IL-6(pg/ml) & $0-7$ & $7.94 \pm 12.98$ & $2.50 \pm 2.83$ & $9.16 \pm 14.04$ & 0.01 \\
\hline Pneumonia & & 56 & 11 & 45 & 0.57 \\
\hline CT(bilateral) & & 36 & 6 & 30 & 0.45 \\
\hline $\mathrm{CT}$ (unilateral) & & 20 & 5 & 15 & \\
\hline
\end{tabular}

Table 4. WBC counts analysis in children and in adults. 


\begin{tabular}{|llllll|}
\hline \multicolumn{5}{|c|}{ Median $(\mathrm{IQR})$} \\
$\mathrm{WBC}\left(\times 10^{9} / \mathrm{L}\right)$ & $4-10$ & $5.17 \pm 1.60$ & $7.04 \pm 2.39 *$ & $3.94 \pm 1.20 \#$ & 0.03 \\
$\mathrm{~N}\left(\times 10^{9} / \mathrm{L}\right)$ & $2-7$ & $3.18 \pm 1.45$ & $1.83 \pm 0.62^{\star *}$ & $1.62 \pm 0.34 \# \#$ & 0.03 \\
$\mathrm{~L}\left(\times 10^{9} / \mathrm{L}\right)$ & $0.8-4$ & $1.57 \pm 0.58$ & $4.67 \pm 1.81^{\star * *}$ & $1.91 \pm 0.93 \# \# \#$ & 0.00 \\
$\mathrm{M}\left(\times 10^{9} / \mathrm{L}\right)$ & $0.12-0.8$ & $0.36 \pm 0.16$ & $0.38 \pm 0.08^{\star * \star *}$ & $0.33 \pm 0.16 \# \# \#$ & 0.80 \\
\hline
\end{tabular}

Note: children $1: \leq 6$; children $2: \varangle 6$, *compared with adults $(p=0.00)$, \#compared with adults $(p=0.13),{ }^{* *}$ compared with adults ( $\left.p=0.01\right)$, \#\#compared with adults $(p=0.02),{ }^{\star \star *}$ compared with adults $(p=0.00)$, \#\#\#compared with adults $(p=0.41)$, ${ }^{\star * \star *}$ compared with adults $(p=0.68)$, \#\#\#\#compared with adults $(p=0.64)$.

Table 5: The proportion of abnormal laboratory tests in children and adults

\begin{tabular}{|lllll|}
\hline & \multicolumn{5}{r|}{$\mathrm{N}(\%)$} & & \\
\hline $\mathrm{WBC}\left(\times 10^{9} / \mathrm{L}\right)$ & Abnormal & Children $(\mathrm{n}=14)$ & Adults(n=53) & $\mathrm{p}$ \\
\hline $\mathrm{N}\left(\times 10^{9} / \mathrm{L}\right)$ & Increased & $0(0 \%)$ & $1(1.9 \%)$ & 0.61 \\
\hline $\mathrm{L}\left(\times 10^{9} / \mathrm{L}\right)$ & Decreased & $1(7.1 \%)$ & $2(3.8 \%)$ & 0.46 \\
$\mathrm{M}\left(\times 10^{9} / \mathrm{L}\right)$ & Increased & $0(0 \%)$ & $5(9.4 \%)$ & 0.79 \\
\hline $\mathrm{HBG}(\mathrm{g} / \mathrm{L})$ & Increased & $1(7.1 \%)$ & $0(0 \%)$ & \\
\hline $\mathrm{PLT}\left(\times 10^{9} / \mathrm{L}\right)$ & Increased & $2(14.3 \%)$ & $16(30.2 \%)$ & 0.08 \\
\hline $\mathrm{CRP}(\mathrm{mg} / \mathrm{L})$ & Increased & $1(7.1 \%)$ & $18(34 \%)$ & 0.048 \\
\hline $\mathrm{PCT}(\mathrm{ug} / \mathrm{L})$ & Increased & $5(35.7 \%)$ & $13(24.5 \%)$ & 0.40 \\
\hline $\mathrm{LDH}(\mathrm{U} / \mathrm{L})$ & Increased & $7(50 \%)$ & $10(18.9 \%)$ & 0.02 \\
\hline $\mathrm{CK}(\mathrm{U} / \mathrm{L})$ & Increased & $4(28.6 \%)$ & $8(15.1 \%)$ & 0.24 \\
\hline $\mathrm{Myo}(\mathrm{ug} / \mathrm{L})$ & Increased & $0(0 \%)$ & $2(3.8 \%)$ & 0.46 \\
$\mathrm{Ctn}(\mathrm{ug} / \mathrm{L})$ & Increased & $0(0 \%)$ & $0(0 \%)$ & \\
\hline $\mathrm{BUN}(\mathrm{mmol} / \mathrm{L})$ & Increased & $0(0 \%)$ & $1(1.9 \%)$ & 0.61 \\
\hline $\mathrm{Cr}(\mathrm{umol} / \mathrm{L})$ & Increased & $0(0 \%)$ & $0(0 \%)$ & 0.14 \\
\hline $\mathrm{PT}(\mathrm{s})$ & Increased & $1(7.1 \%)$ & $7(13.2 \%)$ & 0.53 \\
\hline $\mathrm{D}-\mathrm{dimer}(\mathrm{ug} / \mathrm{ml})$ & Increased & $5(35.7 \%)$ & $19(35.8 \%)$ & 0.99 \\
$\mathrm{ALT}(\mathrm{U} / \mathrm{L})$ & Increased & $1(7.7 \%)$ & $9(17 \%)$ & 0.36 \\
$\mathrm{AST}(\mathrm{U} / \mathrm{L})$ & Increased & $1(7.7 \%)$ & $4(7.5 \%)$ & 0.96 \\
\hline $\mathrm{IL}-6(\mathrm{pg} / \mathrm{ml})$ & Increased & $1(12.5 \%)$ & $12(33.3 \%)$ & 0.24 \\
\hline
\end{tabular}

\section{The younger children patients with COVID-19 had milder clinical symptoms}

There were 8 (57.1\%)asymptomatic cases and 6 (42.9\%)symptomatic cases among the 14 children cases, with a median age of 3.98 years, and 5 cases(62.5\%)were males in the asymptomatic cases and with a median age of 9.17 years, and 1 case(16.7\%)was male in the symptomatic cases. The age of asymptomatic patients was younger than that of symptomatic patients $(p=0.03)($ table6).

Although there were statistically significant differences in white blood cells, monocyte counts, and levels of Cr and AST between asymptomatic and symptomatic children cases, these differences were within the normal range and had no practical clinical significance.

Even among asymptomatic patients, 5(62.5\%)cases had pneumonia including $3(60 \%)$ cases with bilateral pneumonia, with no difference compared with that of asymptomatic cases $(\mathrm{p}=0.58, \mathrm{p}=0.74)$ (table7).

Table 6. Clinical features of COVID-19 in asymptomatic children cases and symptomatic children cases. 


\begin{tabular}{|c|c|c|c|c|}
\hline \multicolumn{5}{|c|}{$N(\%)$} \\
\hline & Total(N=14) & Asymptomatic & Symptomatic & $\mathrm{p}$ \\
\hline & & $\mathrm{N}=8,(57.1 \%)$ & $\mathrm{N}=6,(42.9 \%)$ & \\
\hline Age, median, y & 6.20 & 3.98 & 9.17 & 0.03 \\
\hline \multirow[t]{2}{*}{ Gender } & $M(6,42.9 \%)$ & $M(5,62.5 \%)$ & $\mathrm{M}(1,16.7 \%)$ & 0.09 \\
\hline & $F(8,57.1 \%)$ & $F(3,37.5 \%)$ & $F(5,83.3 \%)$ & \\
\hline Clinical type & 14 & 8 & 6 & 0.09 \\
\hline Mild & $3,(21.4 \%)$ & $3,(37.5 \%)$ & 0 & \\
\hline Conventional & $11,(78.6 \%)$ & $5,(62.5 \%)$ & $6,(100 \%)$ & \\
\hline Severe & 0 & 0 & 0 & \\
\hline Critical & 0 & 0 & 0 & \\
\hline Infection method & 14 & 8 & 6 & \\
\hline Imported & 0 & 0 & 0 & \\
\hline Family cluster & $14,(100 \%)$ & $8,(100 \%)$ & $6,(100 \%)$ & \\
\hline Closed contact & 0 & 0 & 0 & \\
\hline Unclear & 0 & 0 & 0 & \\
\hline Time & $6.18 \pm 6.41$ & $5.43 \pm 6.33$ & $7.17 \pm 9.97$ & 0.64 \\
\hline \multicolumn{5}{|c|}{ Signsandsymptoms } \\
\hline Heart rate & $93 \pm 9.19$ & $93.38 \pm 7.37$ & $92.50 \pm 11.96$ & 0.87 \\
\hline Respiratory rate & $21.67 \pm 1.49$ & $21.54 \pm 1.04$ & $21.83 \pm 2.04$ & 0.73 \\
\hline
\end{tabular}

Note: Time: from onset to diagnosis

Table 7. Lab tests and CT scan of COVID-19 in asymptomatic children cases and symptomatic children cases. 


\begin{tabular}{|c|c|c|c|c|c|}
\hline & \multicolumn{3}{|c|}{ Median (IQR) } & \multirow{3}{*}{$\begin{array}{l}\text { Symptomatic } \\
N=6,(42.9 \%)\end{array}$} & \multirow{3}{*}{$\mathrm{p}$} \\
\hline & NormalRange & Total(N =14) & Asymptomatic & & \\
\hline & & & $\mathrm{N}=8,(57.1 \%)$ & & \\
\hline $\mathrm{WBC}\left(\times 10^{9} / \mathrm{L}\right)$ & $4-10$ & $5.94 \pm 2.52$ & $7.11 \pm 2.38$ & $4.38 \pm 1.85$ & 0.04 \\
\hline $\mathrm{N}\left(\times 10^{9} / \mathrm{L}\right)$ & $2-7$ & $1.76 \pm 0.53$ & $1.93 \pm 0.60$ & $1.53 \pm 0.35$ & 0.17 \\
\hline $\mathrm{L}\left(\times 10^{9} / \mathrm{L}\right)$ & $0.8-4$ & $3.68 \pm 2.04$ & $4.64 \pm 1.77$ & $2.40 \pm 0.1 .73$ & 0.04 \\
\hline $\mathrm{M}\left(\times 10^{9} / \mathrm{L}\right)$ & $0.12-0.8$ & $0.36 \pm 0.11$ & $0.36 \pm 0.09$ & $0.36 \pm 0.14$ & 0.96 \\
\hline $\mathrm{HBG}(\mathrm{g} / \mathrm{L})$ & $110-150$ & $131.64 \pm 12.92$ & $131.63 \pm 9.26$ & $131.67 \pm 17.72$ & 0.10 \\
\hline $\operatorname{PLT}\left(\times 10^{9} / \mathrm{L}\right)$ & $100-300$ & $243.79 \pm 76.16$ & $260.88 \pm 93.38$ & $221.00 \pm 42.20$ & 0.35 \\
\hline CRP(mg/L) & $0.068-8.2$ & $1.26 \pm 2.82$ & $0.65 \pm 0.72$ & $2.08 \pm 4.31$ & 0.46 \\
\hline PCT(ug/L) & $0-0.05$ & $0.05 \pm 0.02$ & $0.05 \pm 0.02$ & $0.05 \pm 0.02$ & 0.69 \\
\hline $\mathrm{LDH}(\mathrm{U} / \mathrm{L})$ & $109-245$ & $298.63 \pm 81.53$ & $333.03 \pm 92.32$ & $252.77 \pm 30.53$ & 0.04 \\
\hline CK(U/L) & $26-140$ & $107.54 \pm 42.95$ & $116.85 \pm 51.82$ & $95.12 \pm 26.70$ & 0.37 \\
\hline Myo(ug/L) & $10-46$ & $15.33 \pm 6.21$ & $16.21 \pm 7.15$ & $14.17 \pm 5.10$ & 0.56 \\
\hline Ctn(ug/L) & $0-0.01$ & $0.01 \pm 0.00$ & $0.01 \pm 0.00$ & $0.01 \pm 0.00$ & \\
\hline $\mathrm{BUN}(\mathrm{mmol} / \mathrm{L})$ & 2.9-8.2 & $4.23 \pm 1.16$ & $4.47 \pm 1.30$ & $3.92 \pm 0.82$ & 0.39 \\
\hline Cr(umol/L) & $50.4-98.1$ & $43.08 \pm 11.19$ & $36.99 \pm 7.04$ & $51.20 \pm 10.86$ & 0.01 \\
\hline PT(s) & $8.8-13.8$ & $12.53 \pm 0.78$ & $12.63 \pm 0.56$ & $12.39 \pm 1.06$ & 0.58 \\
\hline D-dimer(ug/ml) & $0-0.5$ & $0.47 \pm 0.23$ & $0.49 \pm 0.21$ & $0.45 \pm 0.27$ & 0.74 \\
\hline $\operatorname{ALT}(\mathrm{U} / \mathrm{L})$ & $-0-40$ & $21.29 \pm 29.18$ & $28.13 \pm 38.08$ & $12.17 \pm 3.06$ & 0.33 \\
\hline $\mathrm{AST}(\mathrm{U} / \mathrm{L})$ & $0-40$ & $29.00 \pm 8.84$ & $33.38 \pm 7.17$ & $23.17 \pm 7.73$ & 0.03 \\
\hline IL-6(pg/ml) & $0-7$ & $2.50 \pm 2.08$ & $2.00 \pm 0.53$ & $3.17 \pm 3.14$ & 0.32 \\
\hline Pneumonia & & $11,(78.6 \%)$ & $5,(62.5 \%)$ & $6,(100 \%)$ & 0.58 \\
\hline CT(bilateral) & & $6,(54.5 \%)$ & $3,(60 \%)$ & $3,(50 \%)$ & 0.74 \\
\hline CT(unilateral) & & $5,(45.5 \%)$ & $2,(40 \%)$ & 3.(50\%) & \\
\hline
\end{tabular}

\section{Discussion}

The severe acute respiratory syndrome corona virus 2(SARS-CoV-2) infection outbreak has spread in China and around the world ${ }^{[10,11]}$. WHO defined it as a Public Health Emergency of International Concern (PHEIC).Earlier studies found that the initial patients were associated with seafood markets, and the pathogen was soon isolated from the patient's alveolar lavage and identified as anovel coronavirus ${ }^{[2]}$. Coronaviridae (CoVs) are the largest known single stranded RNA viruses. ${ }^{[12]}$ They have been categorized in three groups, alpha-CoVs, beta-CoVs, and gamma-CoVs according to phylogenetic analyses and antigenic criteria. ${ }^{[11]}$ The human Severe Acute Respiratory Syndrome (SARS) virus, the Middle Eastern Respiratory Syndrome (MERS) virus, and theSARS-CoV-2 all belong to beta-CoVs ${ }^{[13]}$. The bat coronavirus (BCoV) and the SARS-CoV-2share $96.2 \%$ sequence identity. Bats were once thought to be the source of SARSCoV-2.However, sufficient evidence is lacking.

Patients with SARS-CoV-2 infection present with a wide range of symptoms. Most patients seem to have mild disease, and about $20 \%$ appear to progress to severe disease, including pneumonia, respiratory failure, and, in some cases, even death ${ }^{[14]}$. However, most of the cases studied were from Wuhan city, and the characteristics of cases in other regions in China, especially in children, have not been reported.

A total of 67 cases including 47 cases from Jinan and 20 cases from Rizhao, of which only 1 case was the severe type and no one was in critical condition, is far lower than reported in Wuhan ${ }^{[15]}$.There were no deaths reported because of few cases of the severe or critical type. The reason for this is considered as follows: 1) There is no major epidemic in the regions except Hubei Province for strong prevention and control and 2) the virulence and pathogenicity of the virus decrease in the $2 \mathrm{nd}$ and 3 rd generation of transmission ${ }^{[16]}$. All cases of children belong to the mild or conventional type of COVID-19, and all children are part of family clusters. Previous studies found no cases in children, who were once thought to be less susceptible ${ }^{[17]}$. According to the current trend, all people including children are susceptible to SARS-CoV-2, and person-to-person transmission develops familial clusters ${ }^{[18]}$. The reason why all children are in family clusters is that it is the traditional Chinese New Year holiday, children have less chance to have contact with the outside world, and Chinese families always attach great importance to the protection of children. 
A retrospective analysis on clinical features of children of COVID-19 compared with that of adults from two research centers was made. The findings showed that clinical symptoms were mild in children with fever and dry cough being the most common symptoms, and other symptoms were rare. However, dry cough and phlegm are not the most common symptoms in children compared with adults.

Previous reports indicated that decreased lymphocytes counts were common clinical features ${ }^{[7,8]}$. However, similar results did not appear in our study, with only a total of 6 cases including 1 case of a child with decreased lymphocytes counts, and the value of lymphocytes counts showed no significant differences in children over 6 years compared with adults. On the other hand, neutrophil counts decreased in children compared with adults( $p=0.02)$. So, lymphocyte decline is not an important indicator for the diagnosis of childhood cases and the neutrophil count decline should be focused on. SARS-CoV-2 infection may induced liver function damage, cardiac muscle damage, kidney damage, and coagulation function changes ${ }^{[7,8]}$. Among these laboratory tests, elevated LDH is more common in children than in adults, which suggested that children are more likely to suffer heart muscle damage. Elevated levels of CRP and IL- 6 showed in adults, but not in children, which should attract more attention. In addition, pneumonia is not uncommon in children and is characterized by bilateral involvement, which is similar to that of adults. However, pneumonia in adults was more severe compared with children(Fig1). Of the 14 children cases, 8 cases were asymptomatic cases. The age of asymptomatic patients was younger than that of symptomatic patients. Even among asymptomatic patients, $62.5 \%$ of cases had pneumonia. Therefore, the lung damage caused by SARS-CoV- 2 is still relatively obvious.

SARS-CoV-2 belongs to lineage B beta coronavirus based on the phylogenetic analysis ${ }^{[19]}$. The spikeprotein (S) of SARS-CoV that mediates entrance to human respiratory epithelial cells by interacting with cell surface receptor angiotensin-converting enzyme 2 (ACE2) is the most important means of pathogenesis ${ }^{[20,21]}$. The number of ACE2 receptors are significantly lower in children than in adults ${ }^{[21]}$, which may be the primary reason why children have fewer clinical symptoms and organ dysfunction than adults. In addition, the weak immune response triggered by viral infection because of children's weakened immune function maybe responsible for this state.

In summary, most cases in children were mild and conventional cases, with mild clinical signs and symptoms, and all were cases of family clusters. Early positive laboratory tests are of little value in diagnosing childhood cases. The possibility of COVID-19 cannot be ruled out by simply relying on the clinical signs, symptoms and positive laboratory tests of children in order to avoid missed diagnosis and delayed treatment.

\section{Abbreviations}

SARS-CoV-2: acute Respiratory Syndrome Coronavirus-2; COVID-19: coronavirus disease 2019;CT: computed tomographic scans; WBC: white blood cell; N: neutrophil; L: lymphocyte; M: monocyte; PLT: blood platelet; CRP: C-reactive protein; PCT: procalcitonin; LDH: lactic dehydrogenase; CK: creatine kinase; Myo: myohemoglobin; Ctn: cardiac troponin; BUN: urea nitrogen; Cr: creatinine; PT: prothrombin time; ALT: glutamic-pyruvic transaminase; AST: glutamic oxalacetic transaminase; IL-6: interleukin-6; ACE2: angiotensin-converting enzyme 2.

\section{Declarations}

\section{Acknowledgement:}

Thanks to Dr. Edward C. Mignot, Shandong University, for linguistic advice.

\section{Contributors}

YJH takes responsibility for the integrity of the data and the accuracy of the data analysis.YJH, WH,ZXG and ZSW had full access to all data in the study. DWJ had the idea of and designed the study. LQ contributed to the review and ZZF approved the final version.

\section{Conflicts of interest}

The authors have no conflicts of interest to disclose.

\section{Funding/support}

None

\section{Ethical Approval}

The project was approved by the ethics board of Jinan infectious hospital (No.20200203).

\section{References}

1.Pneumonia of unknown cause - China', Emergencies preparedness, response, Disease outbreak news, World Health Organization (WHO)

2.Qun Li, Xuhua Guan, Peng Wu,etal.Early Transmission Dynamics in Wuhan, China,of Novel Coronavirus-Infected Pneumonia. N Engl J Med 2020 published online.DOI: 10.1056/NEJMoa2001316

3.WHO.CoronavirusAccessedon20Jan2020. Available at https://www.who.int/health-topics/coronavirus.

4.WHO.CoronavirusAccessedon 11Feb 2020 Available at https://www.who.int/emergencies/diseases/novel-coronavirus-2019

Page $10 / 12$ 
5.http://2019ncov.chinacdc.cn/2019-nCoV/

6. https://www.who.int/csr/don/en

7.ChenN,ZhouM,DongX,etal.Epidemiologicalandclinicalcharacteristicsof99casesof2019novelcoronaviruspneumoniainWuhan,China:adescriptivestudy[publish $\epsilon$ $6736(20) 30211-7$

8.HuangC,WangY,LiX,etal.Clinicalfeaturesofpatientsinfectedwith2019novelcoronavirusinWuhan,China[publishedJanuary24,2020].Lancet.doi:10.1016/S01406736(20)30183-5

9.Lin L, Li TS.Interpretation of "Guidelines for the Diagnosis and Treatment of Novel Coronavirus $\varangle 2019$-nCoV『Infection by the National Health Commission (Trial Version 5)". ZhonghuaYiXue Za Zhi. 2020 Feb 7;100(0):E001.

10.Zhu N, Zhang D, Wang W, et al. A Novel Coronavirus from Patients with Pneumonia in China, 2019[J]. N Engl J Med, 2020, In press. DOI: 10.1056/NEJMoa2001017.1316

11.HuiDS, IAzharE, MadaniTA, et al. The continuing 2019-nCoV epidemic threat of novel coronaviruses to global health: the latest 2019 novel coronavirus outbreak in Wuhan,China[publishedJanuary14,2020].IntJInfectDis.2020;91:264-266.doi:10.1016/j.ijid.2020.01.009

12.J. Cui, F. Li, and Z.-L. Shi, “Origin and evolution of pathogenic coronaviruses," Nat. Rev. Microbiol., vol. 17, no. 3, pp. 181-192, Mar. 2019, doi: 10.1038/s41579-018-0118-9.

13.Carmine Ceraolo and Federico M. Giorgi, Genomic variance of the 2019-nCoV coronavirus, J Med Virol. 2020 Feb 6. doi: 10.1002/jmv.25700.

14.WHO. Novel coronavirus (2019-nCoV) situation reports. Situation report-23. Feb12,2020.https://www.who.int/docs/default-source/coronaviruse/situationreports/20200212-sitrep-23-ncov.pdf?sfvrsn=41e9fb78_2 (accessed Feb 12, 2020)

15.DaweiWang,BoHu,ChangHu etal. Clinical Characteristics of 138 Hospitalized Patients With 2019 Novel Coronavirus-Infected Pneumoniain Wuhan, China. JAMA. Published online February7,2020.doi:10.1001/jama.2020.1585

16.Perlman S, Netland J. Coronaviruses post-SARS: update on replication and pathogenesis. Nat Rev Microbiol 2009; 7: 439-50.

17.Li Q, Guan X, Wu P, et al. early transmission dynamics in Wuhan, China, of novel coronavirus-infected pneumonia. [published on January 29, 2020]. N Engl J Med. 2020.doi:10.1056/NEJMoa2001316

18.ChanJF-W,YuanS,KokK-H,et al. A familial cluster of pneumonia associated with the 2019 novel coronavirus indicating person-to-person transmission :a study of a family cluster [published January 24, 2020]. Lancet. 2020; S0140-6736(20)30154-9. doi:10.1016/S0140-6736(20)30154-9

19.Zhou P, Yang XL, Wang XG, et al. A pneumonia outbreak associated with a new coronavirus of probable bat origin. Nature. 2020. doi:10.1038/s41586-0202012-7.

20.Gallagher TM, Buchmeier MJ. Coronavirus spike proteins in viral entry and pathogenesis. Virology. 2001;279(2):371-374.

21.Wong SK, Li W, Moore MJ, et al. A 193-amino acid fragment of the SARS coronavirus S protein efficiently binds angiotensin-converting enzyme 2. J Biol Chem. 2004;279(5):3197-3201.

\section{Figures}



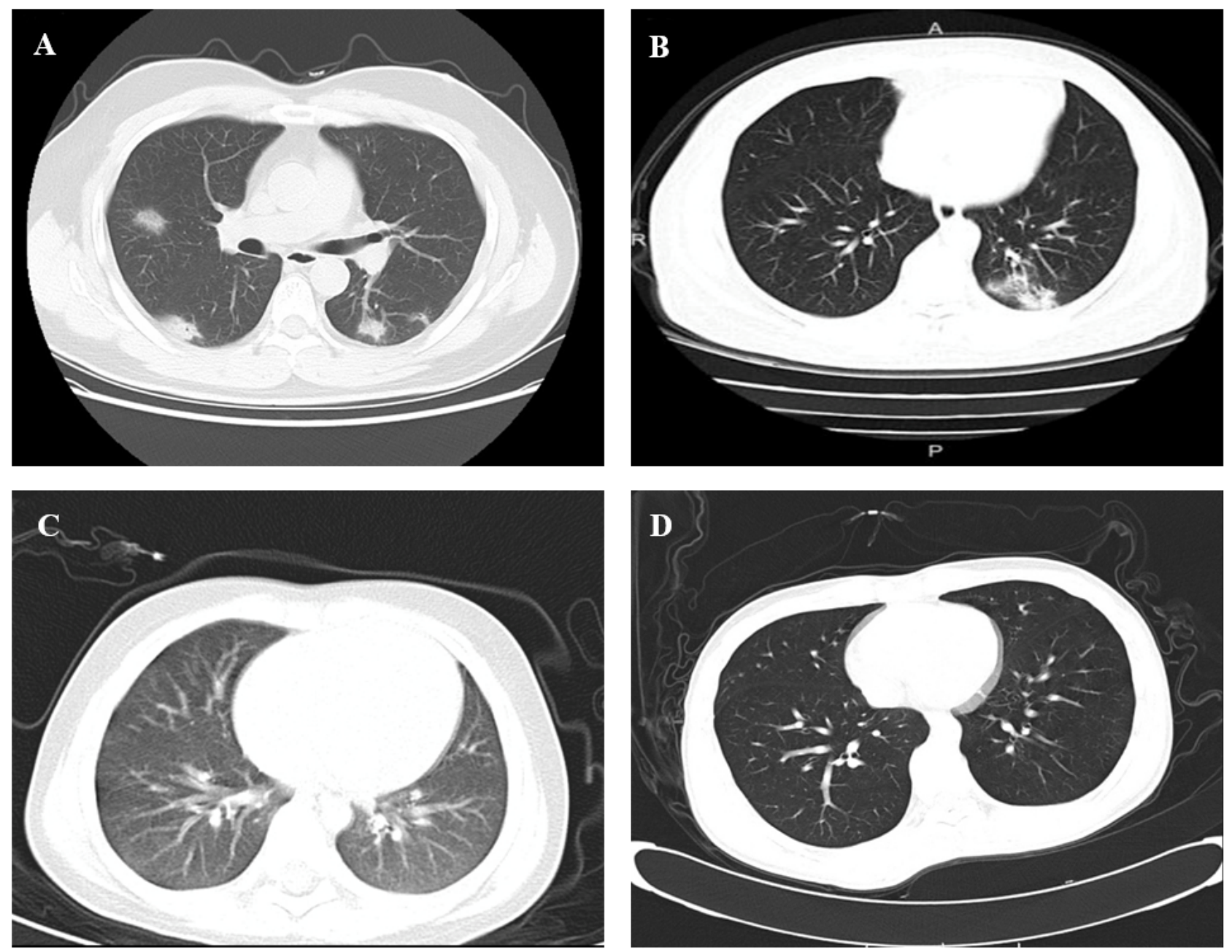

Figure 1

A: Transverse chest CT images from a 42-year-old man showing bilateral multiple lobular and subsegmental areas in the lung on day 7 after symptom onset. B:Transverse chest CT images from a 35-year-old woman showing unilateral lobular and subsegmental areas in the lung on day 5 after symptom onset.

C:Transverse chest CT images from a 5 -year-old male child showing mild bilateral bronchiolitis in the lung on day 5 after symptom onset. D:Transverse chest CT images from a 16-year-old male child showing no obvious lesion in the lung on day 8 after symptom onset. 